\title{
Red social de colaboración de las universidades españolas con in- vestigación en Ingeniería Química
}

\author{
María Isabel Escalona Fernández * \\ Antonio Pulgarín Guerrero \\ María Pilar Lagar Barbosa **
}

Artículo recibido:

25 de mayo de 2010.

Artículo aceptado:

17 de agosto de 2010.

\section{RESUMEN}

El presente trabajo analiza la red social de colaboración científica de las universidades españolas que realizan investigación en el área de "Ingeniería Química”. La red se construye con base en los documentos publicados en el Web of Science durante un periodo de 7 años. Se utiliza el análisis de redes sociales por considerarla como la mejor metodología para conocer la capacidad de colaboración, difusión y transmisión de nuevos conocimientos entre las universidades. Se estudia la cohesión y densidad de la Red, la posición de centralidad de las universidades como principales actores y la posible existencia de subgrupos dentro de

* Universidad de Extremadura, Cáceres, España. escalona@unex.es

** Los dos autores pertenecen a la Universidad de Extremadura, Badajoz, España. (Antonio: pulgarin@unex.es); (María: mplagar@alcazaba.unex.es). 
la red. Los datos se analizan con el software UCINET 6.258 y NetDraw 2.091. Se incorpora al estudio, el índice-H alcanzado por cada una las universidades como indicador de la repercusión y el impacto científico de su investigación.

Palabras Claves: colaboración científica, análisis de redes sociales, universidad española, ingeniería química, índice- $\mathrm{H}$.

\section{ABSTRACT}

Scientific collaboration social network in the field of chemical engineering in spanish universities

María Isabel Escalona Fernández; Antonio Pulgarín Guerrero and María Pilar Lagar Barbosa

This paper analyzes the scientific collaboration social network of Spanish universities within the field of chemical engineering. The network consists of documents published on the Web of Science over a period of seven years. The study employs social network analysis as the most appropriate approach for understanding the capacity for collaboration, dissemination and transfer of knowledge between universities. The paper examines network cohesion and density, the role and degree of centrality of universities in these processes and the existence of sub-groups within the social network. Data gathered was analyzed using the UCINET 6.258 and NetDraw 2.091 software. Each university's $\mathrm{H}$-index score is reported as an indicator of the impact of the research.

Keywords: scientific collaboration, social network analysis, Spanish university, chemical engineering, $\mathrm{H}$-index.

\section{INTRODUCCIÓN}

Dara la "American Institute of Chemical Engineers" (AICHE) la Ingeniería 1 Química es "el campo de la actividad humana en que los conocimientos de las Ciencias Físicas y Naturales, y de la Economía se aplican a fines útiles”. La Ingeniería Química está bien establecida en España y tanto su enseñanza 
como su investigación han progresado sustancialmente en las dos últimas décadas. La industria química española incluye más de 3.700 empresas con unos 135.000 empleados y experimentó un crecimiento del 4\% anual en los primeros años del milenio. La Ingeniería Química es aquella rama de la ingeniería que se dedica al estudio, síntesis, desarrollo, diseño, operación y optimización de todos aquellos procesos industriales que producen cambios físicos, químicos y/o bioquímicos en los materiales. La I Q se fundamenta en las matemáticas, la física y la química, pero sus operaciones se desarrollan sobre la base de los conocimientos aportados por otras ciencias como la biología, la geología, la economía, etcétera, así como por otras ramas de la ingeniería. Este compendio de conocimientos multidisciplinares hace que la colaboración científica adquiera un valor predominante en el desarrollo y avance de esta ciencia. La ingeniería química participa no sólo en la industria química que le es propia sino en otros sectores industriales como la minería, la metalurgia, los materiales de construcción, la tecnología de los alimentos, el tratamiento de vertidos y residuos, la producción de energía, etc. Este campo de aplicación es consecuencia de la interrelación de esta disciplina con otras muchas áreas de la ciencia y de la técnica. Los aspectos relacionados con el medio ambiente constituyen un apartado de particular importancia, no sólo desde el punto de vista de minimizar el impacto ambiental, sino también con la perspectiva de mejorar la eficacia, productividad, seguridad y calidad de los procesos químicos. La ingeniería química se divide en tres grandes sectores: Operaciones básicas; Ingeniería de las reacciones e Ingeniería de procesos.

El papel del ingeniero químico, que es muy productivo en términos de publicaciones, se orienta al estudio del cambio de la composición, o del estado físico o del contenido energético que experimentan los materiales en el proceso industrial.

Son múltiples los motivos que llevan a un investigador a trabajar y publicar en equipo, y tal vez los más plausibles sean la especialización y el aumento de la eficacia del investigador; esto es, la profesionalización (Beaver and Rosen, 1978).

Desde hace varias décadas los responsables en política científica han mostrado un interés creciente por impulsar la colaboración científica entre instituciones, ya que esta mejora tanto la transferencia como la difusión de resultados. Por tanto reforzar la colaboración entre países, dentro de la Unión Europea, como instrumento de cohesión y convergencia viene volviéndose un punto prioritario en política científica (Maltrás y Quintanilla, 1995).

La publicación científica es el vehículo a través del cual se dan a conocer y se difunden los nuevos conocimientos, y por tanto el camino más fácil para 
cuantificar y analizar la colaboración científica entre científicos, universidades, países o áreas de conocimiento.

Price y Beaver (1966) fueron de los primeros investigadores en utilizar las relaciones de coautoría para investigar las estructuras sociales y su influencia en la ciencia, y, más concretamente, en las redes de comunicación.

Price (1963) y Crane (1972) introdujeron y desarrollaron el concepto de "Colegios Invisibles". Diane Crane presentó la idea de "Colegios invisibles" como redes informales de comunicación que tenían alguna forma de organización social. Destacó también la importancia de individuos claves en la difusión de la información a través de todo el campo, y el hecho de que estos individuos eran miembros de "Colegios Invisibles" de élite. Los "Colegios Invisibles" o redes de los autores más productivos son un aspecto importante en la organización social de un área de investigación porque son aquellos que tienden a vincular a los grupos independientes de colaboradores y a promover el desarrollo de un campo.

La colaboración científica contribuye a la creación de conocimiento y su posterior difusión, por ello, el científico o centro de investigación bien posicionado dentro de una red de colaboración recibirá la información más rápidamente y también de mayor calidad (Lambiotte y Panzarasa, 2009).

Desde hace varios años se vienen empleando las redes sociales para analizar la colaboración científica (Jansen, von Gortz, Heidler, 2010; Hou, Kretschmer, Liu, 2008; Valderrama, Gonzalez, Valderrama, Aleixandre, Miguel, 2007). Sin embargo son escasos en esto los estudios sobre un área tan importante para el desarrollo tecnológico y económico del país como es la "Ingeniería Química”.

Peters, Hartmann y Van Raan (1988) describen una forma de llevar a cabo un seguimiento en los avances científicos de la "ingeniería química", determinando los autores de aquellas publicaciones escritas por científicos reconocidos internacionalmente que son más citadas que otras donde escriben científicos menos conocidos. En 1991, Peters y Van Raan llevaron a cabo un análisis de colaboración en varias áreas, e incluyeron a la ingeniería química.

Recientemente se ha propuesto un índice denominado "Índice-H" para valorar la trayectoria investigadora de un científico, que se basa en el número de citas que han recibido sus trabajos (Hirsch, 2005), lo que muestra la solidez y calidad de la investigación de un autor (Van Raan, 2006). El índice-H puede ser aplicado a instituciones o países, y se basa en el número de publicaciones analizadas.

A diferencia de los análisis tradicionales donde los resultados se centran en cuantificar el número de colaboraciones, el análisis de redes sociales permite analizar una "comunidad científica" en su conjunto, valorando a aquellos 
investigadores o instituciones que están mejor relacionados o que poseen mayor influencia sobre el resto de la comunidad.

El análisis de redes sociales (ARS) intenta identificar y analizar la estructura y el comportamiento de una determinada relación social basándose en la teoría de grafos, puesto que es fácil trasladar y aplicar el vocabulario y análisis matemático al estudio de las distintas redes sociales (Wasseman y Faust, 1994). Un grafo está formado por nodos (universidades o países en este estudio) y por líneas que los conectan (colaboraciones en este caso). Las líneas o vínculos que conectan a los distintos nodos pueden ser orientados (en un único sentido) o recíprocos (en ambos sentidos), el primer caso se representa por una flecha con único sentido; y en el segundo, será representado por una flecha con doble punta. Los vínculos en un grafo podrán ser representados de forma binaria o nominal (si existe o no presencia); de forma ordinaria (si el vínculo es más o menos fuerte), o ponderado (según un intervalo o valor promedio).

A través del ARS es posible identificar la estructura general de la red y obtener una medida relativa para su posterior comparación con otras redes. El estudio de la centralidad de la red a través de algoritmos como grado, proximidad o cercanía y mediación, identificarán la posición que ocupan los distintos actores (universidades) o grupos de ellos con respecto al conjunto de la red, o con otros actores. También el ARS detecta los diferentes grupos a los que pertenece un actor (universidad), proporcionando un subgrafo(s) en el cual todos los nodos están conectados entre sí (Hanneman, 2010; Luce y Perry, 1949; Bron y Kerbosch, 1973).

En 1974 Czepiel usó el concepto de centralidad para explicar las pautas de difusión de una innovación tecnológica en la industria del acero, descubriendo que las compañías que ocupaban posiciones más centrales en una red de comunicaciones informales entre empresas, eran las que antes adoptaban un nuevo proceso de fundición. Según Czepiel la centralidad les proporcionaba una ventaja tecnológica.

El objetivo de este trabajo es visualizar y analizar la estructura colaborativa de las universidades españolas que investigan en el área de "Ingeniería Química”, como una red social, observar cómo se interrelacionan unas universidades con otras, y también su comportamiento colaborativo con otros países. Partiendo de la producción científica de las distintas universidades españolas, se pretende estudiar, por un lado, la estructura general de la "comunidad científica” en Ingeniería Química entre universidades, y por el otro la posición que las distintas universidades, o grupo de ellas, ocupan en el conjunto de la comunidad científica. 
El estudio tiene como objetivo analizar la estructura social de la investigación en "Ingeniería Química" de las universidades españolas. Esta estructura social está fundamentada en la colaboración científica que existe entre las distintas universidades.

Para la obtención de los datos se ha considerado como unidad de análisis, la publicación científica; y como fuente de información el Web of Science (WoS) de Thomson Reuters. Se descartó la base de datos SCOPUS de Elsevier por su alto traslapamiento con el Web of Science (Escalona, Lagar y Pulgarín, 2010). En España, gracias a una iniciativa del Ministerio de Ciencia e Innovación la base de datos Web of Science (WoS) es de acceso gratuito para toda la comunidad científica y tecnológica desde el 1 de Enero de 2004; se trata de la base de datos más valorada por todas las instituciones de evaluación de la actividad científica del país.

En la búsqueda de $W o S$ se recuperaron aquellos documentos (artículos y reviews) publicados por investigadores de ingeniería química en España, durante un periodo de tiempo de 7 años.

La estrategia de búsqueda está basada en el campo de la dirección de los autores que aparecen en el campo "address" de WoS (Pulgarín, Escalona y Lagar, en prensa). Los resultados fueron exportados al gestor de bibliografía "Endnote Web". Se analizaron 4.648 documentos publicados entre los años 2000 y 2006, ambos inclusive.

Para el cálculo del índice-H se ordenaron los documentos de cada una de las universidades en orden descendiente de número de citas recibidas, se enumeró e identificó el punto en el que el número de orden coincidía con el de citas recibidas por documento (Hirsch, 2005). De este modo, se obtuvo un índice- $\mathrm{H}$ para cada una de las universidades españolas implicadas en el estudio, independientemente de sus colaboraciones nacionales o internacionales.

Para estudiar la colaboración dentro del área se elaboraron 2 matrices, una con la colaboración entre las universidades (matriz cuadrada de rango 52X52); y otra para la colaboración de las universidades con el exterior (matriz modo-2, formada por 47 universidades y 56 países). Cada celda de la matriz se corresponde con el número de documentos publicados conjuntamente. Cuando un mismo documento ha sido publicado en colaboración con varias instituciones tanto nacionales como internacionales, esta colaboración ya fue cuantificada en cada una de las instituciones o países firmantes en el documento.

En la matriz nacional han sido incorporadas 5 universidades españolas que aunque no tienen un departamento o área específica de investigación en 
"ingeniería química", se presentan como universidades colaboradoras en los documentos analizados, estas universidades son: Universidad Internacional de Cataluña (UIC), Universidad Pompeu Fabra (UPF), Universidad Pontificia de Comillas (UPCO), Universidad San Pablo CEU (UCEU) y la Universidad Nacional de Educación a Distancia (UNED).

Las matrices se analizaron con el software UCINET 6.258 (Borgatti, Everett and Freeman, 2002) y NetDraw 2.091 (Borgatti, 2002).

Para realizar una primera aproximación a la estructura de la red se estudia el nivel de cohesión, analizando su densidad (Doreian, 1974; Burt, 1976), la distancia media entre nodos y el nivel de alcanzabilidad de unas universidades con otras en términos de colaboración científica (Doreian, 1974).

Un análisis de las medidas de centralidad permite conocer la posición de cada universidad dentro de la estructura de la red (Freeman, 1979), para ello se calculan los siguientes indicadores que mostrarán aquellas universidades más centrales, más prestigiosas y con una mayor actividad desde el punto de vista de la colaboración:

Rango o grado nodal (degree): es el número de colaboraciones directas de una universidad; es decir, con cuántas universidades se encuentran directamente conectadas.

Grado de intermediación (betweenness): es la frecuencia con que aparece una universidad en el tramo más corto que conecta a otras dos (Freeman, Borgatti y White, 1991). Este indicador muestra aquellas universidades que son intermediarias entre otras dos del mismo grupo y que nunca han colaborado entre sí. Las universidades con mayor intermediación están aquí en una situación de poder, ya que controlan los flujos de comunicación.

Cercanía (clossenes): mide la distancia media de cada universidad con respecto al resto de universidades de la red.

Se analizan los posibles grupos o subgrafos dentro de la red (cliques). El programa Ucinet utiliza el algoritmo de Bron y Kerbosch (1973) para encontrar todos los cliques o subgrupos dentro de una red. Un Clique es un conjunto de nodos, en nuestro caso universidades, que tienen todos los vínculos posibles entre ellas (Luce y Perry, 1949).

Para la colaboración internacional se obtiene una matriz modo-2 puesto que se analiza la colaboración española hacia el exterior, de ahí que los enlaces entre los nodos vayan en un único sentido (Borgatti y Everett, 1997). 


\section{Red social de colaboración nacional entre universidades españolas}

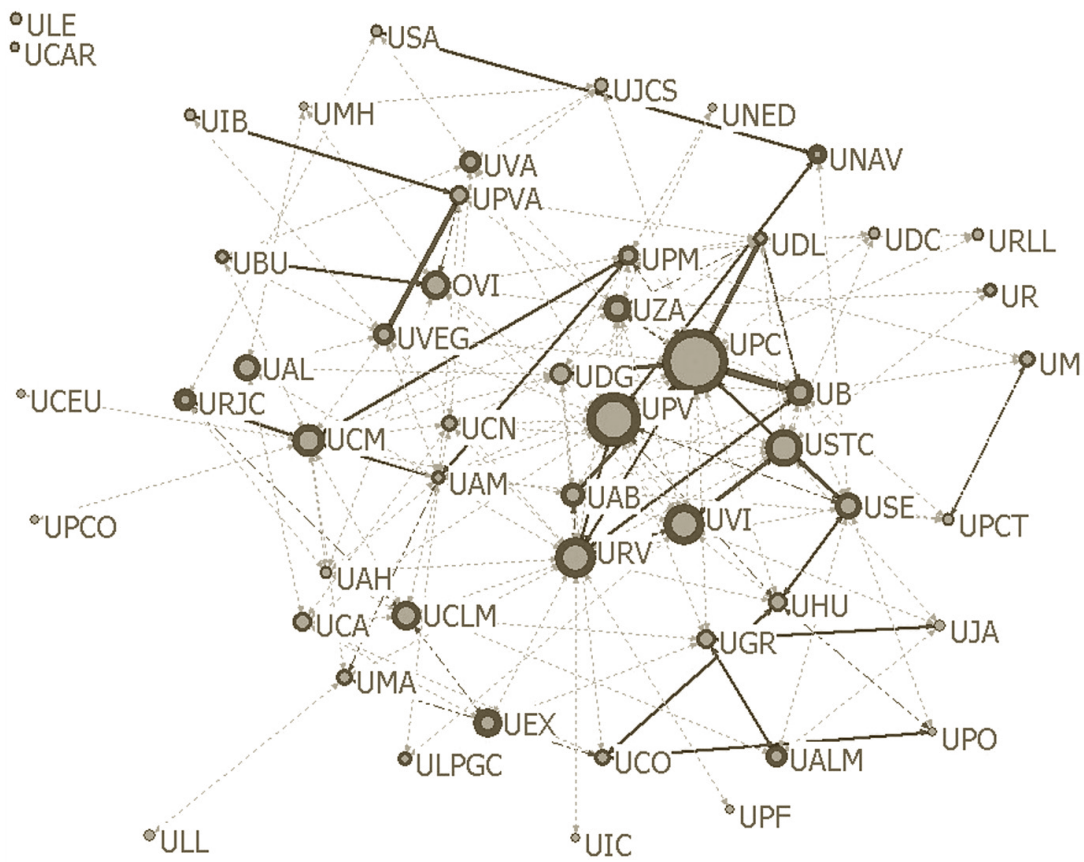

Fig. 1: Red social de colaboración nacional en "Ingeniería Química"

La Figura 1 muestra la representación gráfica de la red social de colaboración nacional en "ingeniería química" de las universidades españolas, donde los nodos representan las distintas universidades y los enlaces muestran el número de colaboraciones, su grosor está en función del número de éstas. Las líneas de puntos muestran aquellas colaboraciones menores a 5; la línea discontinua las colaboraciones entre 5 y 10 y las líneas continuas las colaboraciones mayores a 10. Se han incorporado al nodo de cada universidad, como dos atributos de la red, el número de registros analizados y su índice-H; a mayor número de registros analizados el nodo es más grande, y el grosor de su anillo muestra el Índice$\mathrm{H}$ alcanzado por la universidad en los registros analizados (Anexo I).

Se obtuvieron 3 componentes o subgrafos en los cuales es posible encontrar un camino entre cualquiera de los nodos, 1 de ellos cuenta con 50 nodos y representa un porcentaje de la red del $96,2 \%$; y los otros dos están formados cada uno de ellos por un único nodo aislado, la Universidad de León (ULE) y la Universidad de Cartagena (UCAR) con un porcentaje del 1,9\% cada uno. 
La densidad media de la red mide la proporción de lazos o vínculos existentes en relación con los posibles. Se obtiene así una densidad media de $63,95 \%$. La distancia media (average distance) que existe entre dos nodos mide el esfuerzo que debe hacer una universidad para colaborar con otra, siendo esta distancia de un valor de 2,322, es la distancia media más corta que hay entre dos universidades. El indicador "reachabiblity" indica qué universidades son alcanzables (directa o indirectamente) por otras tomando en cuenta la colaboración. Dentro de la red de colaboración no son alcanzables la Universidad de León y la Universidad de Cartagena; estas dos universidades se muestran dentro de la red como nodos aislados.

\section{Rango o grado nodal (degree)}

El Anexo II muestra las 20 universidades que han alcanzado mayor rango o grado nodal (degree), entre las que destacan, la Universidad Politécnica de Cataluña (UPC), la Universidad de Barcelona (UB), la Universidad Rovira i Virgili (URV) y la Universidad del País Vasco (UPV). Si se observa el rango normalizado (NrmDegree) (Anexo II), la UPC supera con creces el porcentaje de conexiones sobre el total de la red con un $6,112 \%$ de conexiones, y se posiciona dentro de la red como la universidad más conectada dentro del grupo.

La estadística descriptiva muestra información acerca del rango dentro de la red, así, el rango promedio de la red "Mean" es de 32.615 y los valores oscilan entre 240 y 0, el mayor y menor número de colaboraciones.

\section{Intermediación (Betweenness)}

Las universidades con mayor grado de intermediación son La Universidad Rovira i Virgili (URV), La Universidad Complutense de Madrid (UCM), La Universidad Politécnica de Cataluña (UPC) y la Universidad del País Vasco (UPV). La Figura 2 es una representación gráfica de las 10 universidades que presentan un mayor nivel de intermediación. La URV es la universidad con un mayor nivel de intermediación se muestra en una posición de puente entre universidades que no han colaborado nunca juntas. Hay que destacar también la posición de la UB, la que mientras en el grado nodal ocupaba la $2^{a}$ posición como universidad con mayor número de colaboraciones, pasó a ocupar la posición número 14 en niveles de intermediación (Anexo II). 


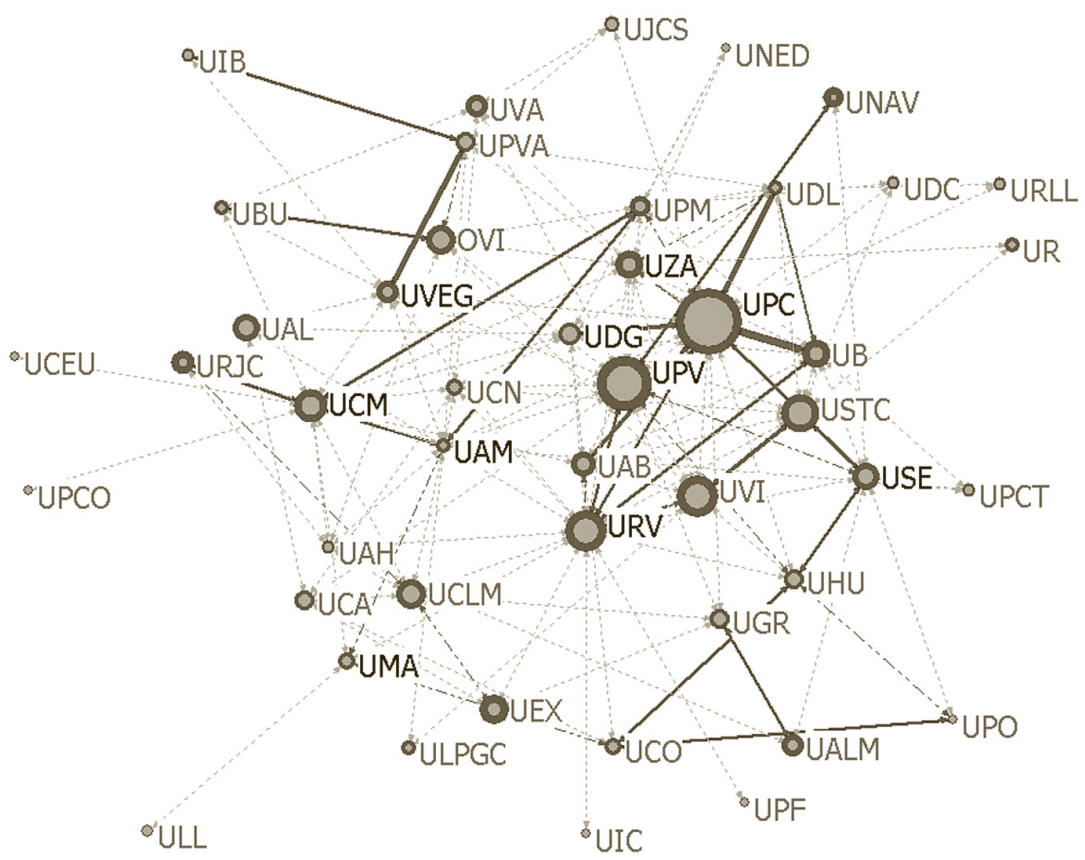

Fig. 2: Red social de colaboración nacional de las 10 Universidades más intermediarias: URV, UCM, UPC, UPV, UAM, UZA, USE, UVEG, UMA, UDG.

\section{Cercanía (Closenes)}

Para conocer la capacidad que tiene una universidad de colaborar con las demás se calcula el grado de cercanía de las distintas universidades con respecto a las demás. Los indicadores mayores sugieren que existe una mayor capacidad de colaboración. Unos valores altos de intermediación junto a valores de cercanía también elevados, hacen pensar en actores (universidades) muy importantes.

En el Anexo II se muestran las medidas de cercanía (Closeness) ordenadas de mayor a menor, en donde las universidades con mayor capacidad de colaboración son la URV, UPC y UPV. Destacan por su posición relevante las universidades UAM y UZA. La Universidad Autónoma de Madrid (UAM) con un grado nodal de 50.000 (posición número 13), pasó a ser la $4^{a}$ en términos de cercanía o capacidad de colaboración con el resto de universidades; la Universidad de Zaragoza (UZA) pasó de la posición número 22 en términos de grado nodal a la posición número 5 en términos de cercanía, y todo ello debido a sus colaboraciones con universidades que tienen una alta centralidad como la Universidad Politécnica de Cataluña (UPC) y la Universidad del País Vasco (UPV). 


\section{Subgrupos (Cliques)}

Evaluando la estructura de la red con un mínimo de 4 componentes se obtienen un total de 21 cliques (Tabla 1), de los cuales 7 de ellos están formados por 5 componentes.

Tabla 1: Cliques localizados con un mínimo de 4 componentes

\begin{tabular}{|l|c|c|c|c|c|c|c|c|c|c|c|c|}
\hline \multicolumn{9}{|c|}{ Cliques encontrados } & & \multicolumn{6}{|c|}{ Cliques encontrados } \\
\hline 1 & UAB & UPC & URV & UVI & UPV & & 12 & USE & UB & URV & USTC & \\
\hline 2 & UHU & UPC & URV & UVI & UPV & & 13 & UZA & OVI & UVI & UPV & \\
\hline 3 & UZA & UPC & URV & UVI & UPV & & 14 & UB & UDG & UDL & UPC & \\
\hline 4 & UHU & USE & UPC & URV & UPV & & 15 & UZA & UB & UDL & UPC & \\
\hline 5 & UPC & URV & UPV & UVEG & & & 16 & UB & UDL & UPC & UPM & \\
\hline 6 & UAB & UB & UDG & UPC & URV & & 17 & UB & UDG & UDL & USTC & \\
\hline 7 & UZA & UB & UPC & URV & & & 18 & UCLM & UAH & UAM & UCM & URJC \\
\hline 8 & USE & UB & UPC & URV & & & 19 & UPC & UAM & UPM & UPV & \\
\hline 9 & UCN & UDG & URV & USTC & & & 20 & UPC & UAM & UPV & UVEG & \\
\hline 10 & UAB & UB & UDG & URV & USTC & & 21 & UPC & UAM & UPVA & UVEG & \\
\hline 11 & UAB & URV & USTC & UVI & & & & & & & & \\
\hline
\end{tabular}

Un análisis de la diagonal de la matriz de superposiciones (Co-Membership Matrix) muestra el número de cliques diferentes a los que pertenece cada universidad (Tabla 2), la UPC junto con la URV son las universidades que forman parte de un mayor número de cliques, 14 y 12 respectivamente, mientras que un total de 30 universidades quedan fuera de los 21 cliques o subgrupos detectados.

Tabla 2: Núm. de cliques a los que pertenece cada universidad.

\begin{tabular}{|c|c|c|c|c|}
\hline Universidades & Núm. de cliqués & & Universidades & Núm. de cliqués \\
\hline UPC & 14 & & USE & 3 \\
\hline URV & 12 & & UVEG & 3 \\
\hline UB & 9 & & UHU & 2 \\
\hline UPV & 8 & & UPM & 2 \\
\hline UDG & 5 & & OVI & 1 \\
\hline USTC & 5 & & UCN & 1 \\
\hline UVI & 5 & & UCLM & 1 \\
\hline UZA & 4 & & UAH & 1 \\
\hline UAB & 4 & & UCM & 1 \\
\hline UDL & 4 & & URJC & 1 \\
\hline UAM & 4 & & UPVA & 1 \\
\hline
\end{tabular}




\section{Red de Colaboración internacional de las universidades españolas}

La Figura 3 muestra la representación gráfica de la Red de Colaboración internacional de las universidades españolas, todas las universidades colaboran a excepción de la Universidad de Illes Balears (UIB) y la Universidad Miguel Hernández de Elche (UMH), ambas universidades se muestran como nodos sueltos dentro de la red.

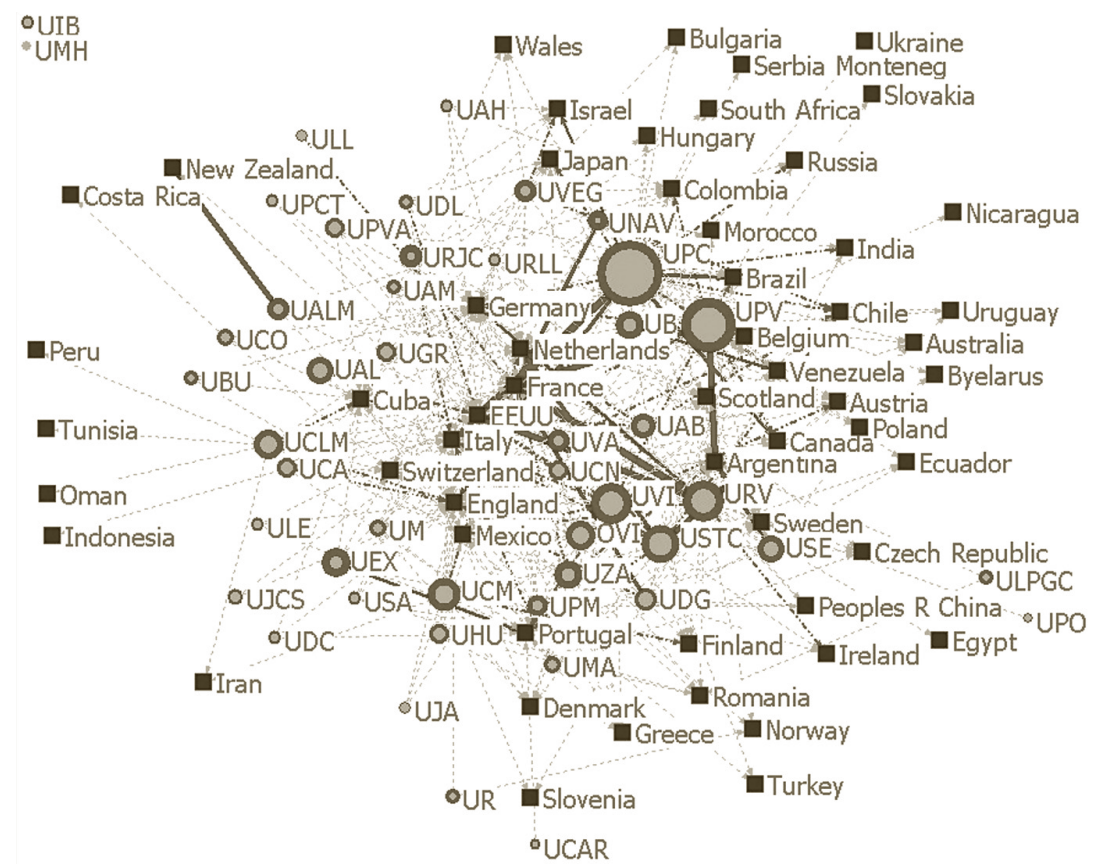

Fig. 3: Red social de colaboración internacional en "Ingeniería Química” de las universidades españolas.

Los nodos representan las distintas universidades y países; y los enlaces el número de colaboraciones, el grosor de las cuales está en función del número de éstas. Al igual que en la colaboración nacional las líneas de puntos muestran aquellas colaboraciones menores a 5; la línea discontinua las colaboraciones entre 5 y 10 y las líneas continuas las colaboraciones mayores a 10. Se han incorporado al nodo de cada universidad el número de registros analizados y su índice-H alcanzado, como dos atributos (Anexo I); a mayor número de registros analizados el nodo es más grande, y el grosor de su anillo está en función del Índice-H obtenido por la universidad en los registros analizados. 
Se obtiene una red con 3 componentes, 2 de ellos formados por una única universidad o nodo aislado, la Universidad de Illes Balears (UIB) y la Universidad Miguel Hernández de Elche (UMH).

\section{Cohesión (2-mode cohesion)}

La Tabla 3 muestra la cohesión de la red de colaboración internacional. La densidad (density) de la matriz es del 15\%, y la distancia geodésica (Avg Dist) es de 2.659. La red está fragmentada ya que es mayor a 0.

Tabla 3: Medidas de Cohesión de la red de colaboración internacional.

\begin{tabular}{|l|l|l|l|l|l|l|}
\hline Density & Avg Dist & Radius & Diameter & Fragmenta & Transitiv & Norm Dist \\
\hline 0.151 & 2.659 & 3.000 & 5.000 & 0.039 & 0.449 & 0.586 \\
\hline
\end{tabular}

\section{Medidas de Centralidad (2-mode centrality)}

Al tratarse de una matriz modo-2, el análisis de la centralidad de la red muestra por separado las medidas de centralidad para las universidades (Anexo III) y los diferentes países (Anexo IV). La Figura 4 muestra las 10 universidades con mayor grado nodal (degreee), entre las que destaca la UCLM con un

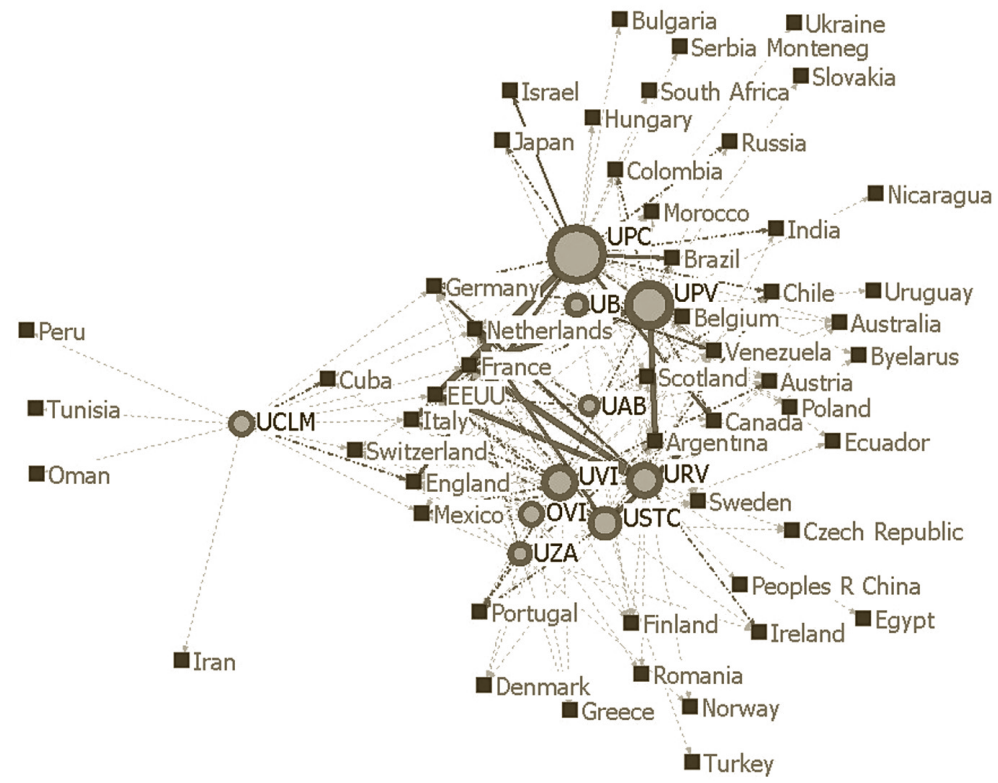

Fig. 4: Red social de colaboración internacional de las 10 universidades con mayor rango o grado nodal: UPC, URV, USTC, UPV, OVI, UAB, UB, UVI, UZA, UCLM. 
alto grado de intermediación (betweenness). La Figura 5 muestra la red de colaboración de los 10 países que alcanzan mayor grado nodal, y se destacan Inglaterra, Alemania, Francia y Estados Unidos.

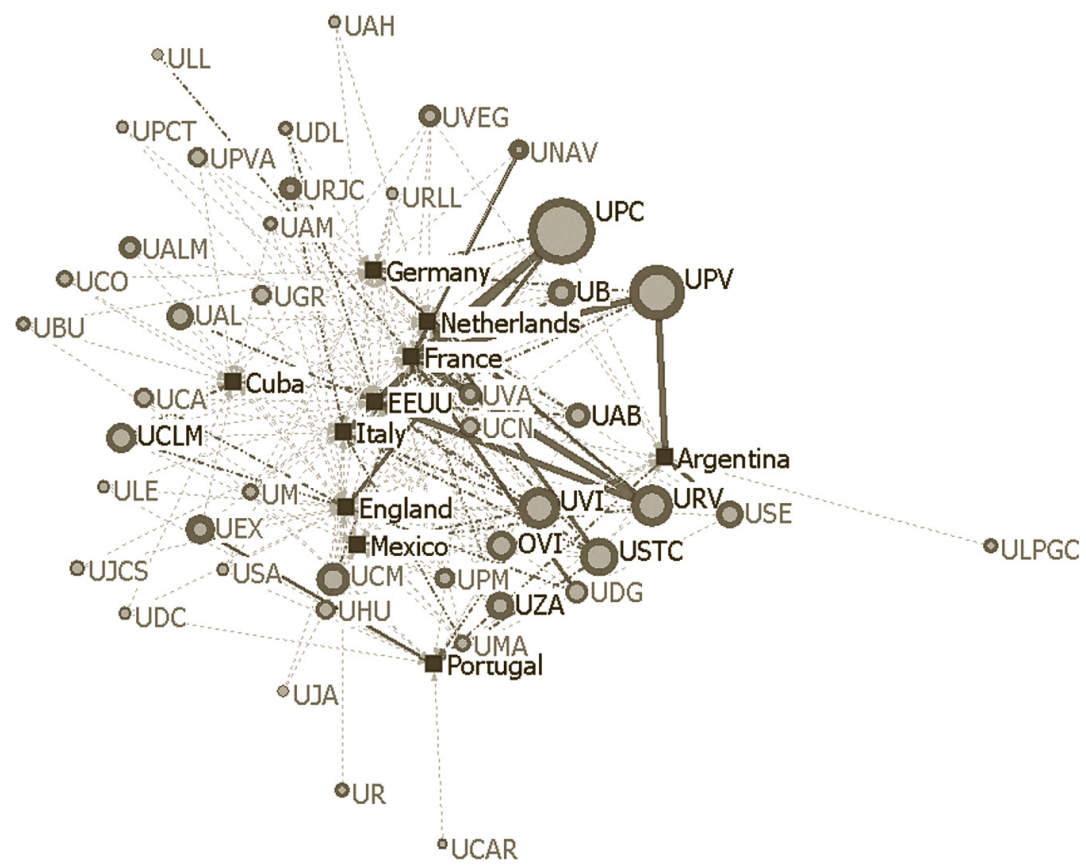

Fig. 5: Red social de colaboración internacional de los 10 países con mayor rango o grado nodal: England, Germany, France, EEUU, Italy, Portugal, Netherlands, Mexico, Argentina, Cuba.

\section{DisCUSIÓN Y CONCLUSIONES}

El objetivo de este trabajo es identificar y analizar la red de colaboración científica de las universidades españolas que investigan en el área de la "Ingeniería Química", aplicando una metodología trasladable a otras áreas de la ciencia que les proporcione a los responsables en política científica un herramienta capaz de potenciar la investigación en un área fundamental para el desarrollo económico de un país. Esta metodología permite identificar las principales universidades y grupos de investigación, dentro de la "Ingeniería Química" en España, y le proporciona también información a las universidades y empresas un punto de referencia y la oportunidad de formar parte de alguno de los grupos o redes destacadas. El estudio toma interés sobre todo porque la industria química española creció alrededor de un $4 \%$ anual a 
principios del milenio, lo que la hizo resaltar como una herramienta útil para desarrollar, mejorar y potenciar esta área de la ciencia.

\section{Colaboración Nacional}

El estudio muestra una universidad española bastante cohesionada en el área de la ingeniería química, pues prácticamente todas las universidades se encuentran, directa o indirectamente, relacionadas. Los resultados muestran a la Universidad Politécnica de Cataluña (UPC) con una posición privilegiada para controlar y difundir la información hacia el resto de las universidades. La Universidad Rovira y Virgili (URV) y la Universidad Complutense de Madrid (UCM) se muestran como universidades puente entre otras que nunca han colaborado entre sí. La metodología pone de manifiesto, además, la importancia de estar bien relacionado, prueba de lo cual son las posiciones alcanzadas por la Universidad Autónoma de Madrid (UAM) y la Universidad de Zaragoza (UZA), las que pese a que el número de sus colaboraciones no es muy alto, están muy bien relacionadas, gracias a sus colaboraciones con la Universidad Politécnica de Cataluña (UPC) y la UPV (Universidad del País Vasco).

De los 21 grupos de colaboración obtenidos 7 están formados por grupos de 5 universidades y el resto por 4. Nuevamente, son la UPC y la URV las universidades que pertenecen al mismo tiempo, a un número mayor de grupos, 14 y 12 respectivamente. De las 52 universidades implicadas en el estudio, 30 no pertenecen a ninguno de los grupos de colaboración existentes, por lo que sería necesario establecer mecanismos de acercamiento de estas universidades con alguno de estos grupos de colaboración.

\section{Colaboración Internacional}

De cara al exterior, la UPC sigue siendo la universidad predominante dentro de la red, en todos sus aspectos de centralidad; es la que mayor número de colaboraciones presenta; es la universidad que se presenta como universidad puente entre otras universidades y la que tiene mayor capacidad de colaboración. Otras universidades que también destacan con una posición relevante dentro de la red social internacional son la Universidad de Santiago de Compostela (USTC) y la Universidad de Castilla la Mancha (UCLM). Países como Inglaterra, Alemania, Francia y Estados Unidos son los preferidos en términos de colaboración por las universidades españolas. También destacan en la red internacional las colaboraciones con países latinoamericanos como México, Argentina y Cuba. 


\section{Índice-H}

Se introduce en el análisis el índice-H alcanzado por cada una las universidades como indicador de la repercusión y el impacto científico de su investigación. Este indicador ha sido calculado en función de los documentos analizados en el periodo de 7 años. Las universidades con mayor visibilidad en términos de centralidad son también las que alcanzan un índice-H más elevado, la UPV, la UPC y la URV con un índice-H de 23, 21 y 21 respectivamente. En este aspecto, destaca la Universidad de Extremadura (UEX) con un índice-H de 20 (ocupando la posición cuarta), sin embargo esta universidad no destaca en términos de centralidad y además no pertenece a ninguno de los 21 grupos de colaboración detectados dentro de la red. Este dato pone de manifiesto la importancia de este estudio como herramienta propiciadora para gestionar e incitar la colaboración científica entre universidades, así como detectar las posibles fortalezas y debilidades de los grupos de investigación.

\section{BiBLIOGRAFÍA}

Beaver, D.; Rosen, R., "Studies in scientific collaboration: Part I-The professional origins of scientific co-authorship", en Scientometrics, v. 1, pp. 65-84, 1978.

Borgatti, S.P., NetDraw: Graph Visualization Software, Harvard, Analytic Technologies, 2002.

Borgatti, S.P.; Everett, M.G., "Network analysis of 2-mode data", en Social Networks, v.19, pp. 243-269, 1997.

Borgatti, S.P.; Everett, M.G.; Freeman, L.C., Ucinet 6 for Windows: Software for Social Network. Analysis, Harvard, Analytic Technologies, 2002.

Bron, C.; Kerbosch, J., "Finding all cliques of an undirected graph", Comm of the ACM v. 16, pp. 575-577, 1973.

Burt, R., "Positions in networks", en Social Forces, v. 55, pp. 93-122, 1976.

Crane, D., The Invisible Colleges: Diffusion of Knowledge in Scientific Communities, University of Chicago Press, Chicago, IL, 1972.

Czepiel, J.A., "Word of mouth processes in the diffusion of a major technological innovation", en Journal of Marketing Research, v. 11, pp.172-180, 1974.

Doreian, P., "On the connectivity of social networks", en Journal of Mathematical Sociology, v. 3, pp. 245-258, 1974.

Escalona-Fernández, M.I.; Lagar-Barbosa, P.; Pulgarín-Guerrero, A., "Web of Science vs. Scopus: un estudio cuantitativo en ingeniería química”, en Anales de Documentación, v. 13 (en prensa), 2010. 
Freeman, L.C., "Centrality in Social Networks: Conceptual clarification", en Social Networks, v.1, pp. 215-239, 1979.

Freeman, L.C.; Borgatti, S.P.; White, D.R., "Centrality in valued graphs: A measure of betweenness based on network flow", en Social Networks, v. 13, pp. 141-154, 1991.

Hanneman, R.A., "Introduccion a los métodos de análisis de redes sociales”, Departamento de Sociología de la Universidad de California Riverside, versión en castellano en red: http://www.redessociales.net [consultado el 5 de Mayo de 2010].

Hirsch, J.E., "An index to quantify an individual's scientific research output", en Proceedings of the National Academy of Sciences of the United States of America, v. 102, núm. 46 pp. 16569-16572, 2005.

Hou, H.; Kretschmer, H; Liu, Z., "The structure of scientific collaboration networks in Scientometrics", en Scientometrics, v. 75, núm. 2, pp. 189-202, 2008.

Jansen, D.; von Gortz, R.; Heidler, R., "Knowledge production and the structure of collaboration networks in two scientific fields", Scientometrics, v. 83, núm 1, pp. 219-241, 2010.

Lambiotte, R.; Panzarasa, P., "Communities, knowledge creation, and information diffusion”, en Journal of Informetrics, v. 3, núm. 3, pp. 180-190, 2009.

Luce, R.; Perry, A., "A method of matrix analysis of group structure", en Psychometrika, v.14, pp. 95-116, 1949.

Maltrás, B.; Quintanilla, M.A., Indicadores de la producción científica: España, 1986-1991, Madrid, Consejo Superior de Investigaciones Científicas, 1995.

Peters, H.P.F.; Van Raan, A.F.J., Co-word Based Maps of Chemical Engineering, Research Report to the Netherlands Foundation for Technological Research (STW), Research Report CWTS-91-03, Centre for Science and Technology Studies, Leiden, (116 pp), 1991.

Peters, H.P.F.; Hartmann, D.; Van Raan, A.F.J. "Monitoring Advances in Chemical Engineering”, en Egghe, L.; Rousseau, R. (Editors), Informetrics 87/88, Elsevier Science Publishers, Amsterdam, pp. 175-195, 1988.

Price, D.J. de Solla; Beaver, D., "Collaboration in an invisible college”, en American Psychologist, v. 21, pp. 1011-1018, 1966.

Price, D.J. de Solla, Little Science, Big Science, New York, Columbia Universty Press, 1963.

Pulgarín-Gerrero, A.; Lagar-Barbosa, M.P.; Escalona-Fernández, M.I., "Colaboración científica de la ingeniería química en las universidades españolas", en Revista General de Información y Documentación (Aparecerá).

Valderrama-Zurian, J.C.; González-Alcaide, G.; Valderrama-Zurian, F.J., Aleixandre-Benavent, R.; Miguel-Dasit, A., "Coauthorship networks and institutional collaboration in Revista Española De Cardiologia publications", en Revista Española de Cardiología, núm. 60, v. 2, pp. 117-130, 2007. 
Van Raan, A.F.J., "Comparison of the Hirsch-index with standard bibliometric indicators and with peer judgment for 147 chemistry research groups", en Scientometrics, p. 67, núm. 3, pp. 491-502, 2006.

Wasserman, S.; Faust, K., Social Network Analysis: Methods and Applications, Cambridge: Cambridge University Press, 1994. 


\section{ANEXOS}

Anexo I. Acrónimos de las Universidades Españolas, índice-Hy Número de registros analizados.

\begin{tabular}{|c|c|c|c|}
\hline & Universidades & Índice-H & Número de registros \\
\hline $\mathrm{OVI}$ & Universidad de Oviedo & 13 & 183 \\
\hline UAB & Universidad de Autónoma de Barcelona & 13 & 122 \\
\hline UAH & Universidad de Alcalá & 5 & 20 \\
\hline UAL & Universidad de Alicante & 15 & 138 \\
\hline UALM & Universidad de Almería & 13 & 79 \\
\hline UAM & Universidad Autónoma de Madrid & 8 & 39 \\
\hline UB & Universidad de Barcelona & 16 & 140 \\
\hline UBU & Universidad de Burgos & 8 & 39 \\
\hline UCA & Universidad de Cádiz & 10 & 77 \\
\hline UCAR & Universidad Carlos III de Madrid & 5 & 13 \\
\hline UCLM & Universidad de Castilla-La Mancha & 15 & 163 \\
\hline UCM & Universidad Complutense de Madrid & 18 & 213 \\
\hline UCN & Universidad de Cantabria & 8 & 73 \\
\hline UCO & Universidad de Córdoba & 9 & 70 \\
\hline UDC & Universidad de A Coruña & 7 & 38 \\
\hline UDG & Universidad de Girona & 9 & 105 \\
\hline UDL & Universidad de Lleida & 8 & 41 \\
\hline UEX & Universidad de Extremadura & 20 & 154 \\
\hline UGR & Universidad de Granada & 9 & 93 \\
\hline UHU & Universidad de Huelva & 10 & 88 \\
\hline UIB & Universidad de Illes Balears & 7 & 22 \\
\hline UJA & Universidad de Jaén & 3 & 17 \\
\hline UJCS & Universidad Jaume I de Castellón & 7 & 60 \\
\hline ULE & Universidad de León & 7 & 28 \\
\hline ULL & Universidad de La Laguna & 4 & 26 \\
\hline ULPGC & Universidad de Las Palmas de Gran Canaria & 9 & 43 \\
\hline UM & Universidad de Murcia & 8 & 58 \\
\hline UMA & Universidad de Málaga & 10 & 61 \\
\hline UMH & Universidad Miguel Hernández de Elche & 1 & 5 \\
\hline UNAV & Universidad Pública de Navarra & 17 & 52 \\
\hline UPC & Universidad Politécnica de Cataluña & 21 & 583 \\
\hline
\end{tabular}




\begin{tabular}{|c|c|c|c|}
\hline & Universidades & Índice-H & Número de registros \\
\hline UPCT & Universidad Politécnica de Cartegena & 6 & 30 \\
\hline UPM & Universidad de Politécnica de Madrid & 12 & 77 \\
\hline UPO & Universidad Pablo de Olavide & 3 & 11 \\
\hline UPV & Universidad del País Vasco & 23 & 448 \\
\hline UPVA & Universidad Politécnica de Valencia & 9 & 100 \\
\hline UR & Universidad de La Rioja & 8 & 33 \\
\hline URJC & Universidad Rey Juan Carlos & 17 & 94 \\
\hline URLL & Universidad Ramón Llull & 5 & 16 \\
\hline URV & Universidad Rovira i Virgili & 21 & 303 \\
\hline USA & Universidad de Salamanca & 6 & 30 \\
\hline USE & Universidad de Sevilla & 15 & 149 \\
\hline USTC & Universidad de Santiago de Compostela & 17 & 272 \\
\hline UVA & Universidad de Valladolid & 13 & 80 \\
\hline UVEG & Universidad de Valencia Estudi General & 14 & 99 \\
\hline UVI & Universidad de Vigo & 19 & 302 \\
\hline UZA & Universidad de Zaragoza & 17 & 141 \\
\hline
\end{tabular}

Anexo II. Medidas de centralidad de la red social de colaboración nacional de las 20 universidades con mayor grado nodal (degree).

\begin{tabular}{|c|c|c|c|c|c|c|c|c|}
\hline & Degree & NrmDegree & & Betweenness & nBetweenness & & Farness & nCloseness \\
\hline UPC & 240.000 & 6.112 & URV & 217.778 & 17.081 & URV & 186.000 & 27.419 \\
\hline UB & 116.000 & 2.954 & UCM & 152.661 & 11.973 & UPC & 188.000 & 27.128 \\
\hline URV & 90.000 & 2.292 & UPC & 148.685 & 11.662 & UPV & 189.000 & 26.984 \\
\hline UPV & 75.000 & 1.910 & UPV & 114.164 & 8.954 & UAM & 195.000 & 26.154 \\
\hline UCM & 73.000 & 1.859 & UAM & 100.042 & 7.846 & UZA & 196.000 & 26.020 \\
\hline UPVA & 68.000 & 1.732 & UZA & 72.245 & 5.666 & UCM & 198.000 & 25.758 \\
\hline UDL & 62.000 & 1.579 & USE & 64.238 & 5.038 & UDG & 198.000 & 25.758 \\
\hline UVI & 60.000 & 1.528 & UVEG & 59.830 & 4.693 & USTC & 200.000 & 25.500 \\
\hline USTC & 60.000 & 1.528 & UMA & 58.733 & 4.607 & UVI & 200.000 & 25.500 \\
\hline UVEG & 58.000 & 1.477 & UDG & 56.744 & 4.451 & UAB & 200.000 & 25.500 \\
\hline USE & 54.000 & 1.375 & UPM & 56.305 & 4.416 & UCN & 201.000 & 25.373 \\
\hline UDG & 54.000 & 1.375 & USTC & 52.955 & 4.153 & UB & 201.000 & 25.373 \\
\hline UAM & 50.000 & 1.273 & UVI & 45.414 & 3.562 & UVEG & 202.000 & 25.248 \\
\hline UPM & 46.000 & 1.171 & UB & 40.691 & 3.191 & UPM & 204.000 & 25.000 \\
\hline
\end{tabular}




\begin{tabular}{|c|c|c|c|c|c|c|c|c|}
\hline & Degree & NrmDegree & & Betweenness & nBetweenness & & Farness & nCloseness \\
\hline UAB & 46.000 & 1.171 & OVI & 39.626 & 3.108 & USE & 204.000 & 25.000 \\
\hline UHU & 46.000 & 1.171 & UAH & 29.711 & 2.330 & UCLM & 207.000 & 24.638 \\
\hline UCO & 43.000 & 1.095 & UCLM & 29.267 & 2.295 & OVI & 208.000 & 24.519 \\
\hline UNAV & 42.000 & 1.070 & UVA & 28.589 & 2.242 & UAH & 208.000 & 24.519 \\
\hline UGR & 36.000 & 0.917 & UCN & 25.292 & 1.984 & UHU & 209.000 & 24.402 \\
\hline OVI & 35.000 & 0.891 & UPVA & 24.580 & 1.928 & UPVA & 211.000 & 24.171 \\
\hline
\end{tabular}

Anexo III. Medidas de centralidad de la red social de colaboración internacional de las 20 universidades con mayor grado nodal (degree).

\begin{tabular}{|c|c|c|c|c|c|c|c|}
\hline & Degree & & Closeness & & Betweenness & & Eigenvector \\
\hline UPC & 0.536 & UPC & 0.740 & UPC & 0.144 & UPC & 0.322 \\
\hline URV & 0.446 & URV & 0.712 & URV & 0.102 & URV & 0.294 \\
\hline USTC & 0.357 & USTC & 0.685 & UPV & 0.076 & USTC & 0.275 \\
\hline UPV & 0.339 & UPV & 0.673 & UCLM & 0.072 & UAB & 0.251 \\
\hline OVI & 0.304 & OVI & 0.667 & USTC & 0.057 & UVI & 0.248 \\
\hline UAB & 0.286 & UVI & 0.655 & UB & 0.044 & OVI & 0.243 \\
\hline UB & 0.286 & UAB & 0.649 & OVI & 0.033 & UPV & 0.243 \\
\hline UVI & 0.286 & UB & 0.649 & UCM & 0.032 & UB & 0.211 \\
\hline UZA & 0.232 & UZA & 0.643 & UZA & 0.024 & UZA & 0.196 \\
\hline UCLM & 0.232 & UCM & 0.643 & UVI & 0.024 & UCM & 0.194 \\
\hline UCM & 0.232 & UCLM & 0.627 & UCA & 0.023 & UCLM & 0.185 \\
\hline UVA & 0.196 & UVA & 0.617 & UGR & 0.022 & UVA & 0.172 \\
\hline UVEG & 0.196 & UGR & 0.612 & UAB & 0.022 & UM & 0.143 \\
\hline UGR & 0.179 & UCA & 0.607 & UCO & 0.020 & UCA & 0.139 \\
\hline UMA & 0.179 & UDG & 0.607 & UVEG & 0.020 & UMA & 0.137 \\
\hline UDG & 0.179 & UMA & 0.602 & UMA & 0.016 & URJC & 0.137 \\
\hline USE & 0.161 & UPM & 0.602 & UDG & 0.016 & UGR & 0.135 \\
\hline UCN & 0.161 & URJC & 0.602 & UPM & 0.014 & UDG & 0.135 \\
\hline URLL & 0.161 & UM & 0.602 & UVA & 0.013 & USE & 0.132 \\
\hline UPM & 0.161 & UVEG & 0.602 & URJC & 0.013 & UCN & 0.129 \\
\hline
\end{tabular}


Anexo IV. Medidas de centralidad de la red social de colaboración internacional de los 20 países que presentan mayor grado nodal (degree).

\begin{tabular}{|l|c|l|c|l|c|l|c|}
\hline & Degree & & Closeness & & Betweennes & & Eigenvector \\
\hline England & 0.617 & England & 0.822 & England & 0.115 & England & 0.343 \\
\hline Germany & 0.532 & Germany & 0.797 & France & 0.087 & Germany & 0.323 \\
\hline France & 0.511 & France & 0.789 & Germany & 0.087 & France & 0.320 \\
\hline USA & 0.511 & USA & 0.789 & USA & 0.084 & USA & 0.306 \\
\hline Italy & 0.447 & Italy & 0.766 & Italy & 0.077 & Italy & 0.281 \\
\hline Portugal & 0.383 & Netherlands & 0.730 & Portugal & 0.045 & Netherlands & 0.238 \\
\hline Netherlands & 0.362 & Mexico & 0.686 & Netherlands & 0.042 & Portugal & 0.223 \\
\hline Mexico & 0.298 & Argentina & 0.674 & Argentina & 0.030 & México & 0.200 \\
\hline Argentina & 0.277 & Cuba & 0.657 & México & 0.028 & Argentina & 0.181 \\
\hline Cuba & 0.277 & Portugal & 0.657 & Sweden & 0.028 & Switzerland & 0.172 \\
\hline Switzerland & 0.255 & Canada & 0.641 & Cuba & 0.026 & Canada & 0.156 \\
\hline Sweden & 0.234 & Venezuela & 0.641 & Switzerland & 0.016 & Sweden & 0.151 \\
\hline Belgium & 0.191 & Belgium & 0.636 & Belgium & 0.008 & Venezuela & 0.151 \\
\hline Brazil & 0.191 & Brazil & 0.636 & Brazil & 0.008 & Belgium & 0.147 \\
\hline Canada & 0.191 & Sweden & 0.636 & Canada & 0.008 & Brazil & 0.139 \\
\hline Venezuela & 0.191 & Switzerland & 0.631 & Colombia & 0.008 & Cuba & 0.139 \\
\hline Greece & 0.170 & Scotland & 0.625 & Venezuela & 0.008 & Austria & 0.126 \\
\hline Scotland & 0.170 & Colombia & 0.621 & Morocco & 0.007 & Finland & 0.120 \\
\hline Austria & 0.149 & Austria & 0.611 & Romania & 0.007 & Scotland & 0.113 \\
\hline Colombia & 0.149 & Morocco & 0.611 & Scotland & 0.007 & Denmark & 0.111 \\
\hline
\end{tabular}

